\title{
La cristallographie, fondement essentiel des sciences et des technologies
}

Décidée par l'Organisation des Nations Unies, I'Année internationale de la cristallographie 2014 laisse le devant de la scène à l'Année internationale de la lumière (IYL2015). La cristallographie est entrée dans le champ scientifique un siècle avant que sa lumière de prédilection, les rayons $X$ - suivis dans la seconde moitié du $20^{\mathrm{e}}$ siècle par les électrons et les neutrons -, permette son développement dans toutes les sciences. Essaimant rapidement au-delà de la physique et de la chimie avec le soutien essentiel des mathématiques, elle est un des piliers de la biologie, de la métallurgie, de la science des matériaux, verres et polymères compris, des sciences de la Terre et de la médecine.

Portée initialement par les minéralogistes - dont René Just Haüy (1743-1822) - séduits par la beauté des gemmes et des cristaux et la symphonie des symétries géométriques, la cristallographie irrigue d'une manière déterminante toutes les filières scientifiques et économiques. De la microélectronique à la pharmacie, des semi-conducteurs cristaux quasi parfaits avec des « défauts contrôlés » savamment dosés qui déterminent les propriétés d'usage, à l'ADN, double hélice biologique présente dans toutes les cellules et porteuse de l'information génétique, les recherches et les découvertes ultérieures dans toutes les disciplines se construisent sur la connaissance de l'organisation géométrique des atomes et groupes d'atomes dans la matière inerte ou vivante.

À Paris, le 19 janvier 2015, lors de la séance inaugurale d'IYL2015 à l'UNESCO, Jaurès Alferov, prix Nobel de physique 2000 pour ses travaux en physique et technologie des hétérostructures semi-conductrices, rappelait l'inanité et la nocivité de la séparation, entretenue par certains milieux de décideurs court-termistes, entre recherche fondamentale et recherche appliquée. La vision d'Alferov est claire : toutes les découvertes sont appliquées, porteuses d'une rupture ou d'une évolution incrémentale exploitable aujourd'hui, ou présentes sur les étagères, patiemment à l'affût, pour être croisées avec d'autres découvertes et entrer dans le cycle économique.

"Non, mille fois non, il n'existe pas une catégorie de sciences auxquelles on puisse donner le nom de sciences appliquées, il y a une science et les applications de la science, liées entre elles comme le fruit et l'arbre qui l'a porté. » Ainsi répétait Louis Pasteur, docteur ès sciences pour ses travaux en cristallographie et sur l'importance de l'asymétrie, marque de la vie, profondément convaincu de la fertilisation croisée des sciences avec les technologies.

À la suite du rapport national sur l'impact socio-économique des mathématiques en France, présenté au MENESR par Cédric Villani le 27 mai 2015, le journal Le Monde introduisait ainsi l'événement : "Cette fois, c'est prouvé, les maths sont vraiment utiles. Cette discipline aux multiples facettes contribuerait même pour 15\% du PIB national, soit 285 milliards d'euros, et $9 \%$ des emplois, soit 2,1 millions de personnes. Ce nombre croît de 0,9\% par an, contre 0,5\% pour l'ensemble des emplois. " (Étude de l'impact socio-économique des mathématiques en France, réalisée par le cabinet de conseil en stratégie CMI.)

II me semble pertinent de rappeler dans ce contexte l'analyse économique indépendante, commandée par la Société Européenne de Physique (EPS) en 2012 au cabinet Cebr (Centre for Economics and Business Research). Elle couvre 29 pays européens et provient des données statistiques publiques d'Eurostat. La physique a créé plus de 15 millions d'emplois hautement qualifiés en Europe entre 2007 et 2010, soit 13\% de l'emploi directement productif. De chacun d'eux découlent 2,7 nouveaux emplois (www.eps.org/resource/resmgr/policy/EPS_economyExSummary2013.pdf).

Fuyons le travers de notre temps où il faut classer tout ce qui existe en introduisant des évaluations quantitatives dénuées de tout sens. Mathématiques et physique cultivent, et c'est heureux, des champs économiques communs. Rappelé par tous les évènements de l'année internationale, le croisement interdisciplinaire porté par la cristallographie est d'une telle ampleur, d'une telle diversité, que toute approche quantitative de son impact socio-économique est hors de portée.

Dans ce contexte, il est nécessaire de continuer à offrir dans l'enseignement supérieur une formation à - et par la cristallographie. Elle est aujourd'hui et demain, indispensable à de nombreux champs scientifiques et technologiques, nourris par des mathématiques élégantes, porteuses de concepts profondément intriqués avec ceux de la minéralogie, de la chimie, de la physique... et de la biologie.

- Les approches structurales des protéines, des empilements épitaxiés à deux, voire une, dimensions de la microélectronique et des nanosciences, ont produit de nouveaux chapitres de la cristallographie. Les microscopes électroniques, la RMN, le rayonnement synchrotron, porteurs de nouvelles techniques expérimentales, ont permis de voir plus intimement l'organisation structurale locale, prolongeant la vision de la diffraction et de la spectroscopie des RX de laboratoire, des neutrons et des électrons.

- La cristallographie n'est pas une science achevée : les espaces de dimension supérieure à trois ont ouvert des territoires où ses nouvelles extensions ont fait émerger des concepts originaux et résolu des structures complexes mais ordonnées, comme les modulations incommensurables et les quasicristaux.

- Toutes les productions industrielles sont dépendantes de la cristallographie et des caractérisations qu'elle a permis d'implanter, de la découverte et optimisation des matériaux à la réalisation des produits des grands secteurs économiques. Les plus visibles ces dernières décennies sont ceux de l'industrie pharmaceutique, qui utilise beaucoup le rayonnement synchrotron.

Alain Fontaine

Président de la Société Française de Physique 